\title{
Straw biochar strengthens the life strategies and network of rhizosphere fungi in manure fertilized soils
}

\author{
Dandan Wang ${ }^{1,2}$, Na Zhang ${ }^{1,3}$, Haoqi Tang ${ }^{1}$, Jonathan M. Adams ${ }^{1}$, Bo Sun ${ }^{1}$, Yuting Liang ${ }^{1, *}$ \\ 1 State Key Laboratory of Soil and Sustainable Agriculture, Institute of Soil Science, Chinese Academy of Sciences, Nanjing 210008, China \\ 2 School of Environmental and Safety Engineering, Changzhou University, Changzhou 213164, China \\ 3 University of the Chinese Academy of Sciences, No.19A Yuquan Road, Beijing 100049, China
}

\section{ARTICLE INFO}

\section{Article history:}

Received June 19, 2018

Revised October 18, 2018

Accepted October 23, 2018

Keywords:

Rhizosphere

Biochar

Fungal community

Life strategies

Network analysis

\section{A B S T R A C T}

Soil fungi have many important ecological functions, however, their life strategies and interactions in manure fertilized soils are not well understood. The aim of this study was to investigate the effects of biochar amendment on the fungal life strategies and species interactions in ryegrass (Lolium perenne L.) rhizosphere soil by high-throughput sequencing. Three soil treatments were evaluated: soil and pig manure mixture without planting ryegrass and biochar application (bulk soil), mixture with ryegrass planting (rhizosphere soil (RS)), and addition of $2 \%(\mathrm{w} / \mathrm{w})$ biochar with ryegrass (RS + biochar). Our results indicated that temporal turnover, defined as the slope of linear regression between community similarity and time, was significantly higher in the biochar amendment (slope $=-0.2689, p<0.0001$ ) relative to the rhizosphere soil. Following biochar addition, the percentage of species employing slow acclimation ecological strategies decreased (from $27 \%$ to $17 \%$ ) and the percentage of sensitive species increased (from $40 \%$ to $50 \%$ ) in comparison to the rhizosphere soil. Network analysis indicated that fungal communities in the biochar amendment enhanced positive correlations compared to the rhizosphere soil and bulk soil. Structural equation model indicated that soil $\mathrm{pH}$ was the most important factor in altering fungal life strategies and interactions in manure fertilized soils.

(c) Higher Education Press 2019

\section{Introduction}

Fungi are important members of soil microbial communities, as they secrete numerous enzymes that degrade organic compounds. Fungi can decompose complex organic compounds more efficiently than bacteria do and thus play an important role in soil carbon cycling (Sun et al., 2016). Moreover, fungi are highly associated with crops to form mycorrhizae, which greatly facilitate plant growth and maintain the stability of agricultural ecosystems (Liu et al., 2015).

\footnotetext{
* Corresponding author

E-mail address: ytliang@issas.ac.cn (Y. Liang)
}

Biochar is usually applied as a soil amendment for improving soil environment, increasing soil fertility and/or adsorbing soil pollutants (Jia et al., 2018). Biochar is reported to influence soil fungal biomass, community structures, functions, and enzyme activities through both direct and indirect ways (Steinbeiss et al., 2009; Durenkamp et al., 2010; Ameloot et al., 2013). Direct mechanisms include: (i) biochar acts as habitat for fungi (Lehmann et al., 2011), and (ii) biochar contains nutrients that promote fungal growth (Yamato et al., 2006). Indirect mechanisms include: (i) biochar changes soil nutrient availability by affecting physical and chemical environments, thereby indirectly influencing soil fungi (Warnock et al., 2007), and (ii) biochar is beneficial to other microbial populations, such as mycorrhization helper bacteria 
and phosphate solubilizing bacteria, which then indirectly affects fungal growth (Warnock et al., 2007). However, the succession and the underlying mechanisms of fungal communities (especially, the rhizosphere fungi) in response to biochar addition have not been fully elucidated.

Life strategy is the combination of population characteristics that correspond to the microbial living environment, which reflects the ecological choice and strategy of the population (Evans and Wallenstein, 2014). As was proposed in 1977 for plant taxa (Grime, 1977), we can similarly divide microbial taxa into broad categories on the basis of shared life-history strategies. Using this framework to assess variability in life strategies within soils, various microbial community functions exhibiting complex structures can be explored from an ecological perspective. Further, the richness of soil nutrient content can be evaluated from a microbiological point of view. By adopting this approach, we can then predict key traits for major taxa and better understand microbial influence on soil processes (Fierer, 2017). Moreover, in complex ecosystems like soil environments, the interactions between populations are more important to the function of the ecosystem than their richness and diversity (Deng et al., 2012). Network analysis techniques can provide insight into potential interactions within complex microbial communities (Gilbert et al., 2012), and the topological properties of the network can provide insight into the stability and functional diversity of the community (Dickie et al., 2016). Ecological network analysis methods have recently been used to investigate interactions between species in large microbial communities, and to identify microbial ecological networks (Holland and Hastings, 2008; Fuhrman, 2009). Here, these analytical methods are used to investigate the life strategies and interactions among rhizosphere fungal communities.

The current work focused on the responses and mechanisms underlying the diversity, life strategies, and interactions of soil fungal populations and further assessed the relationship between soil environments and fungal community. The objectives of this study were as follows: (i) understand the diversity and successive variation in rhizosphere fungal communities with biochar amendment, (ii) investigate the effects of biochar on the life strategies and interactions of rhizosphere fungal populations, and (iii) identify the primary factors that affect life strategies and interactions among rhizosphere fungal populations.

\section{Materials and methods}

\subsection{Experimental design and soil sampling}

On the basis of a survey on antibiotic distribution in pig manure and in soil samples in early stages of our experiment, fresh pig manure samples were collected from a pig farm in Changzhou, Jiangsu $\left(31^{\circ} 44^{\prime} \mathrm{N}, 119^{\circ} 45^{\prime} \mathrm{E}\right)$, and surface soil samples $(0-10 \mathrm{~cm})$ without antibiotics were taken from the experimental paddy field of the pig farm. The samples were stored on ice and transported to the laboratory. Any visible living plant materials (e.g., roots) were manually removed from the soils in the laboratory. Soil and pig manure samples were air-dried, sieved through a $2 \mathrm{~mm}$ mesh, and then evenly mixed (100:1 w/w soil:pig manure). The mixture was allowed to stand for $24 \mathrm{~h}$, stirred, and then allowed to stand again for another $24 \mathrm{~h}$. Finally, the soils were placed in plastic pots at $3 \mathrm{~kg} \cdot \operatorname{pot}^{-1}$.

A total of 81 experimental pots were used in this experiment, 27 pots with ryegrass planting, 27 pots with ryegrass and $2 \%(\mathrm{w} / \mathrm{w})$ biochar amendment, and 27 pots with manure only as bulk soil. In general, 30 seeds were planted per pot. At $0,5,10,15,20,25,30,35$, and 40 days after germination, ryegrass was gently removed to separate the root system. The shake-off method was then used to collect rhizosphere soils. Triplicate pots were taken was replicates. Lolium perenne seeds were obtained from the Forage Research Institute of the Jiangsu Academy of Agricultural Sciences. Biochar samples were prepared by pyrolysis and carbonization of wheat stalks at $650^{\circ} \mathrm{C}$ under anoxic conditions. The sample geochemical variables were measured per sampling day, including: soil $\mathrm{pH}$, nitrate $\left(\mathrm{NO}_{3}{ }^{-} \mathrm{N}\right)$, ammonium nitrogen $\left(\mathrm{NH}_{4}{ }^{+}-\mathrm{N}\right)$, available phosphorous, trace elements ( $\mathrm{Si}$, $\mathrm{Al}, \mathrm{Fe}, \mathrm{K}, \mathrm{Ca}, \mathrm{Ti}, \mathrm{S}, \mathrm{Zr}, \mathrm{Mn}, \mathrm{V}, \mathrm{Sr}, \mathrm{Zn}, \mathrm{Cr}, \mathrm{Rb}, \mathrm{Y}$ and $\mathrm{Nb}$ ), soil surface structural parameters (the surface area, micropore area, external surface area and micropore volume), and ryegrass root surface characteristics (the total length, total diameter, total surface area, total cross-sectional area, average length, average diameter, average surface area and average cross-sectional area). In addition, since the large amounts of residual antibiotics in manure fertilized soil can alter the fungi activity and community structures in soils (Hammesfahr et al., 2008), therefore, antibiotic concentrations were also determined from the collected rhizosphere soils, including chlortetracycline, doxycycline, tetracycline, sulfamethazine, enrofloxacin, lomefloxacin, ciprofloxacin and norfloxacin (Liang et al., 2017).

\subsection{Illumina sequencing analysis of ITS2 gene amplicons}

The microbial genomic DNA was extracted from $2 \mathrm{~g}$ of wellmixed soil for each sample by Power Soil DNA isolation kit (MoBio Laboratories, Inc., Carlsbad, CA, U.S.A) according to the manufacturer's instructions. The extracted DNA quality was determined using a Nanodrop 2000 (Thermo Fisher Scientific, Wilmington, DE, U.S.A) according to the $260 / 280$ $\mathrm{nm}$ and $260 / 230 \mathrm{~nm}$ absorbance ratios. All DNA was stored at $-80^{\circ} \mathrm{C}$. The primers ITS3F (5'-GCATCGATGAAGAACGCAGC-3') and ITS4R (5'-TCCTCCGCTTATTGATATGC-3') were used to amplify the ITS2 region, which is the universal DNA barcode marker for the molecular identification of fungi (Op De Beeck et al., 2014). Both forward and reverse primers were tagged with adapter, pad and linker sequencing. A unique barcode was added to the reverse primer to permit the multiplexing of samples.

PCR amplification was performed in triplicate in a $20 \mu \mathrm{L}$ mixture for each reaction and included 1 reaction buffer (NEB $\left.\mathrm{Q}^{\mathrm{TM}}\right), 2 \mathrm{mM} \mathrm{Mg}^{2+}, 0.2 \mathrm{mM} \mathrm{dNTP}, 0.1 \mu \mathrm{M}$ of each primer, $1 \mathrm{U}$ 
Q5 ${ }^{\mathrm{TM}}$ DNA polymerase and $2 \mu \mathrm{L}$ template DNA. Thermal cycling conditions were as follows: an initial denaturation at $94^{\circ} \mathrm{C}$ for $2 \mathrm{~min}$, followed by 25 cycles of $94^{\circ} \mathrm{C}$ for $30 \mathrm{~s}, 55^{\circ} \mathrm{C}$ for $30 \mathrm{~s}$ and $72^{\circ} \mathrm{C}$ for $1 \mathrm{~min}$, with a final extension at $72^{\circ} \mathrm{C}$ for 10 min, paused at $4^{\circ} \mathrm{C}$. To add sample-specific index sequence, a $20 \mu \mathrm{L}$ mixture for each reaction and included $1 \times$ reaction buffer (NEB Q5 ${ }^{\mathrm{TM}}$ ), $0.3 \mathrm{mM}$ dNTP, $0.25 \mu \mathrm{M}$ of $\mathrm{F}$ primer, 0.25 $\mu \mathrm{M}$ of index primer, $1 \mathrm{U} 5^{\mathrm{TM}} \mathrm{DNA}$ polymerase and $1 \mu \mathrm{L}$ diluted template. The cycling program started at $94^{\circ} \mathrm{C}$ for 2 min followed by 8 cycles of $94^{\circ} \mathrm{C}$ for $30 \mathrm{~s}, 55^{\circ} \mathrm{C}$ for $30 \mathrm{~s}, 72^{\circ} \mathrm{C}$ for $1 \mathrm{~min}$ and final $72^{\circ} \mathrm{C}$ for $10 \mathrm{~min}$, paused at $4^{\circ} \mathrm{C}$. Illumina libraries were constructed using the MiSeq Reagent Kit v2 according to manufacturer's instructions. High-throughput, paired-end sequencing was performed on the Illumina MiSeq PE250 platform at Center for Genetic and Genomic Analysis, Genesky Biotechnologies Inc., Shanghai, China, according to standard protocols.

The raw data obtained from Illumina sequencing were analyzed as follows: (i) removed any low quality or ambiguous reads (reads with lengths $<200 \mathrm{bp}$, ambiguous bases, primer mismatches, or average quality scores < 15); (ii) OTUs were defined as sequences at the $97 \%$ similarity level; (iii) taxonomy assignment of fungal OTUs was conducted in Mothur (version 1.39.3) using the command "classify.seqs." (https://www.mothur.org/wiki/Classify.seqs) with a bootstrap support of $60 \%$ against UNITE version 7.1 reference databases (https://unite.ut.ee/); (iv) the ITS2 gene sequence was subjected to a similarity search one by one against the GenBank nucleotide database (nt) provided by NCBI using the BLASTN algorithm (http://www.ncbi.nlm.nih.gov); and (v) calculated alpha-diversity indices (Chao, Ace, Simpson and Shannon) to assess the internal (within-sample) complexity of individual microbial populations.

\subsection{Statistical analysis}

Regular analysis of variance (ANOVA) was implemented to measure the differences among treatments. Alpha diversity was calculated using Shannon index. The number of OTUs shared by or specific to the various treatments was conducted with a Venn diagram using the website Venny (Venny 2.1.0). The fungal distribution patterns of different treatments were determined by nonmetric multidimensional scaling (NMDS), and estimated by a permutation analysis of variance (PERMANOVA). This was performed in the $\mathrm{R}$ software package (version 3.2.5) using the vegan library. The timedecay relationship was plotted as $\ln$ (community similarity) against In(time) and a linear regression performed to obtain the slope. The beta diversity of soil fungal communities was analyzed using the Bray-Curtis distance method by vegan in $R$ (Korhonen et al., 2010). To examine the significance of timedecay relationship curves, we tested whether those slopes were significantly less than zero with two-tailed test by bivariate correlations in the SPSS software packages (version 20.0). Also, the significance of slopes among three treatments was tested by same method. Heat map was drawn using pheatmap in $\mathrm{R}$.
Network analysis was used to investigate changes in the associations among fungal communities in soil. All statistical methods for the network analysis were conducted using the Co-occurrence Network inference (CoNet) plugin for Cytoscape (version 3.4.0). The fungal OTUs data matrix of three treatments and an OTU annotation file were prepared in the formats as guided in the CoNet. The OTUs were filtered by setting 15 as the minimum occurrence value across 27 samples for each treatment. Then, we selected four measures to calculated network relationships: Pearson correlation, Spearman correlation, Bray-Curtis similarity and KullbackLeibler divergence. The initial positive and negative edge numbers were set at 1000 . For each measure and edge, 1000 renormalized permutation and 1000 bootstrap scores were computed to mitigate compositionality bias. The measurespecific $p$-value was computed first and then merged with Brown's method. Edges with merged $p$-values below 0.05 were kept after multiple testing using the Benjamini-Hochberg procedure (Xiao et al., 2017). The final network was obtained after the permutations as the null distribution and the bootstraps as the random distribution. NetworkAnalyzer was used to analyze the resulting network in terms of topological parameters. The network was visualized and customized based on the type of relationships between OTUs and topological network parameters. Key nodes were classified by their topological properties implemented in the extremevalues package in $\mathrm{R}$. The functional group (guild) of the OTUs was inferred using FUNGuild v1.0, an open annotation tool for parsing fungal community data sets by ecological guild independent of sequencing platform (Nguyen et al., 2016).

The Mantel and partial Mantel tests were used to calculate the correlations between environmental factors and the soil fungal community, which were implemented by $R$ package ecodist. Structural equation model (SEM) was carried out to evaluate the direct and indirect relationships between ryegrass root, soil $\mathrm{pH}$, trace element, soil structure, antibiotics, fungal life strategies and interactions in AMOS 22.0.0 using maximum likelihood.

\subsection{Accession number}

The Illumina sequence reads were deposited in the Sequence Read Archive (SRA) at the NCBI under accession number PRJNA392841.

\section{Results}

\subsection{Fungal community patterns}

A total of 75 fungi communities were assessed with sequencing and the number of sequences obtained per sample ranged from 44941 to 98438 . To minimize the impact of read count variation among samples, samples were rarefied based on the sample with the lowest read count (44941 per sample). The DNA contents revealed no significant differences among treatments except on day 35 (Fig. S1(a)). The number of OTUs per sample ranged from 373 to 591 in the bulk soil, from 
375 to 595 in the rhizosphere soil (RS), and from 341 to 591 in the RS + biochar (Fig. S1(b)). We did observe that species richness varied over time. RS + biochar exhibited higher fungal richness compared to bulk soil, with the exception of day 10. Fungal a-diversity (as indicated by the Shannon index) increased with time (Fig. S1(c)). The number of OTUs that were detected also varied across the three treatments, as indicated by a Venn diagram (Fig. S1(d)). Fifty-seven OTUs were shared among the three treatments. The relative abundance of dominant taxa changed distinctly in the different treatments (Fig. S2).

A non-metric multidimensional scaling ordination plot was used to analyze changes in fungal community structure over time (Fig. 1). The close clustering of samples from the beginning of the experiments reflected the similar origins for the soils and consequently, similar fungal community compositions. Fungal community compositions were significantly different among treatments (PERMANOVA, pseudo- $F=$ 4.558, $p<0.001)$.

\subsection{Temporal turnover of fungal communities}

We evaluated the decay of fungal community similarities over time (time-decay) by In-linear regression modeling (Fig. 2). The rate of temporal change in fungal community structure is the slope of the model, and the slope is thus a measure of fungal community turnover (Shade et al., 2013). To assess time-decay in these fungal communities, we used a similar approach as Shade et al. (2013). A significant time-decay relationship was observed for the fungal communities within all three treatment groups, which exhibited negative slopes of: $-0.2042(p<0.0001)$ for the bulk soil, $-0.1161(p<0.0001)$ for the RS and $-0.2689(p<0.0001)$ for the RS + biochar. The slope of the biochar amendment was significantly $(p<0.0001)$ steeper than the rhizosphere soil.

The fungal time-decay relationships among taxonomic divisions were also estimated (Table 1). Considerable variation in slope values were observed for different phyla. In addition, the Ascomycota and Chytridiomycota phyla exhibited significantly $(p<0.0001)$ steeper slopes in the RS + biochar compared to the bulk soil. These results indicate that the biochar amendment accelerated the temporal turnover ( $\beta$ diversity) of rhizosphere fungal communities at the phylum level.

\subsection{Shifts in fungal life strategies}

The species in the bulk soil, RS and RS + biochar were divided into three life strategy groups on the basis of different response patterns among treatments (Fig. 3). The three life strategies were identified as follows: i) slow acclimating (exhibiting low relative abundances in the earlier stage $(0-$ $10 \mathrm{~d})$, low after the middle stage (15-25 d), and high after the later stage (30-40 d)), ii) fast acclimating (low, high, and high, in each experimental stage, respectively), and iii) sensitive (high, low, and low, in each experimental stage, respectively). After filtering the OTU data set to exclude rare and lowabundance sequences, a total of 1581,1678 , and 1743 OTUs were identified from the bulk soil, RS, and RS + biochar, respectively. In addition, we identified 590, 591, and 611 species in the three treatments, respectively (Fig. S3).

Compared to the bulk soil, the percentage of species employing sensitive strategies was only $40 \%$ in the RS (from $44 \%$ in the bulk soil), while the percentage of fast acclimating species increased from $29 \%$ in the bulk soil to $33 \%$ in the RS. However, the percentage of species employing slow acclima-

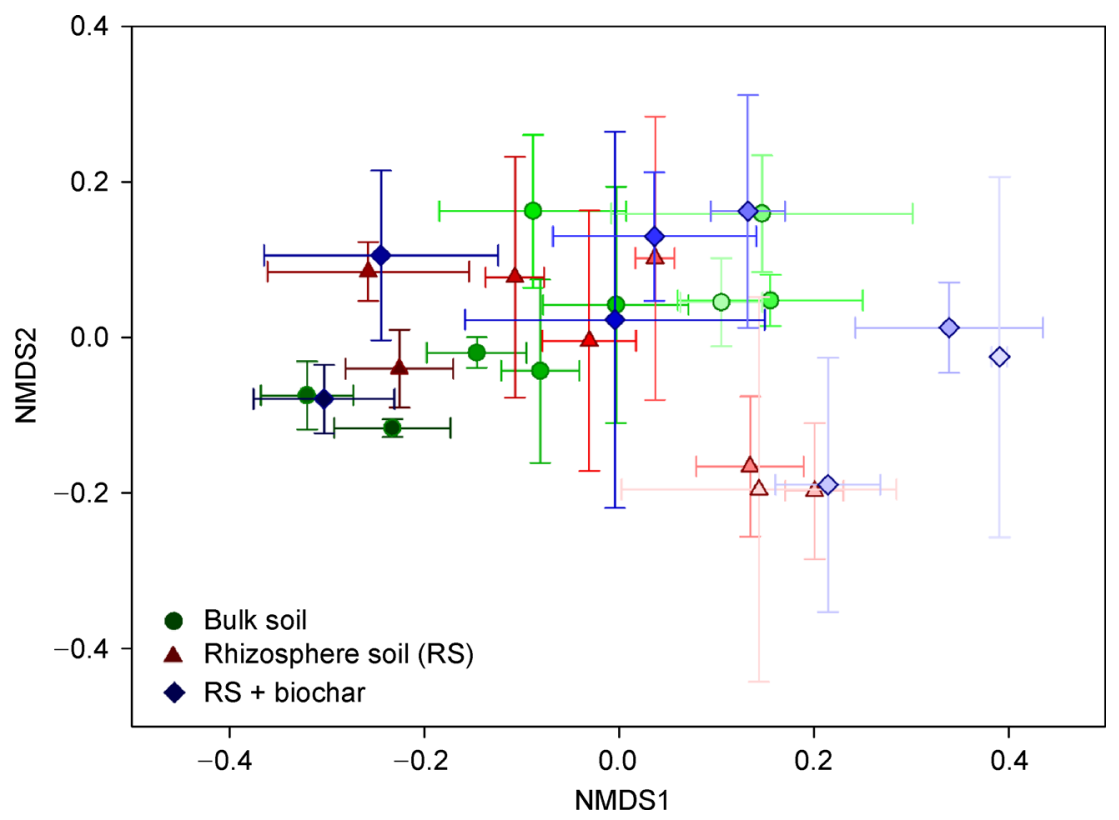

Fig. 1 Non-metric multidimensional scaling (NMDS) analysis of the soil sample. Error bars represent the standard deviation and the color from deep to shallow represents the fungal community succession from the beginning to the end of the experiment ( 40 days). 


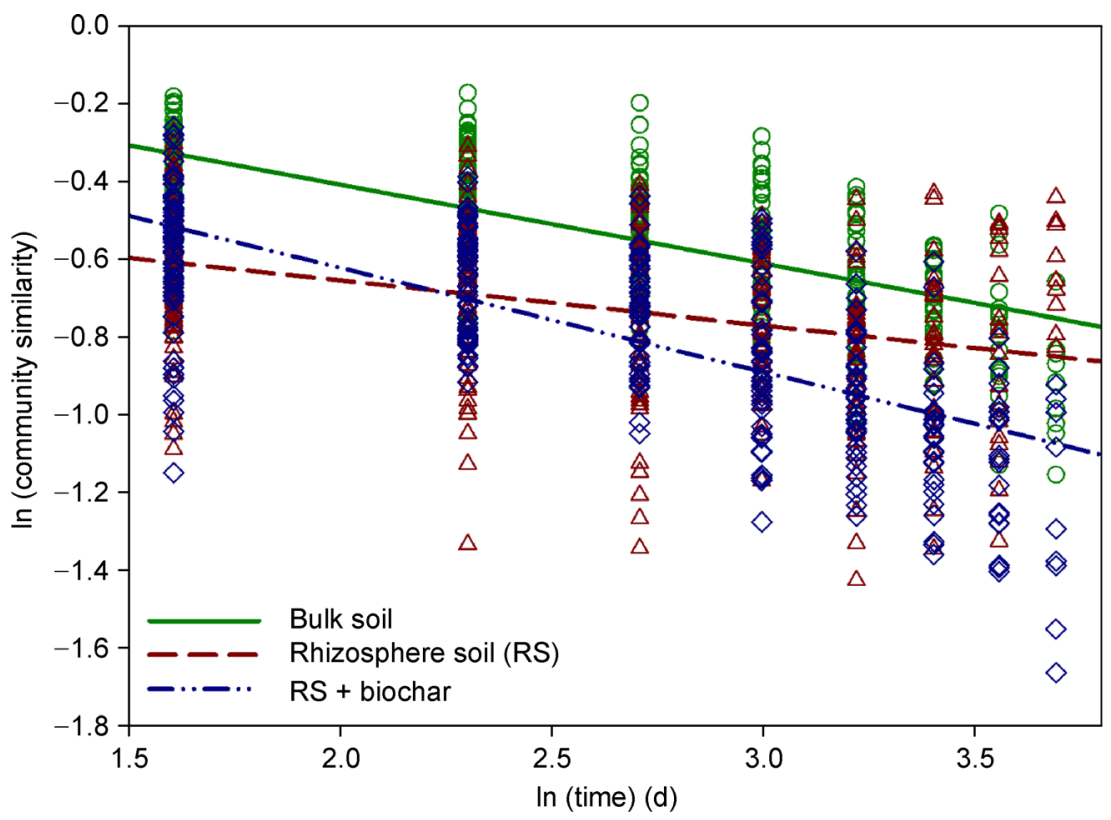

Fig. 2 Time-decay curves for the soil fungal communities, the slopes of all the lines are significantly less than zero.

Table 1 Temporal turnover (slope values) of the fungal communities among different phylogenetic groups.

\begin{tabular}{|c|c|c|c|c|c|c|c|c|c|}
\hline & \multicolumn{3}{|c|}{ Bulk soil } & \multicolumn{3}{|c|}{ Rhizosphere soil (RS) } & \multicolumn{3}{|c|}{ RS + biochar } \\
\hline & Slope & $R^{2}$ & $p$ & Slope & $R^{2}$ & $p$ & Slope & $R^{2}$ & $p$ \\
\hline ITS all & -0.2042 & 0.4739 & $<0.0001$ & -0.1161 & 0.1218 & $<0.0001$ & -0.2689 & 0.485 & $<0.0001$ \\
\hline Ascomycota & -0.0034 & 0.0027 & 0.354 & 0.0012 & 0.0002 & 0.796 & -0.033 & 0.1103 & $<0.0001$ \\
\hline Basidiomycota & 0.0163 & 0.0048 & 0.216 & -0.0246 & 0.0018 & 0.445 & -0.0435 & 0.0119 & 0.050 \\
\hline Chytridiomycota & -0.2859 & 0.1246 & $<0.0001$ & -0.1487 & 0.0353 & 0.001 & -0.3132 & 0.1013 & $<0.0001$ \\
\hline Zygomycota & -0.0391 & 0.013 & 0.041 & 0.0581 & 0.0126 & 0.043 & -0.0319 & 0.0022 & 0.401 \\
\hline Unclassified_fungi & -0.0869 & 0.035 & 0.001 & -0.1328 & 0.0796 & $<0.0001$ & -0.3578 & 0.2479 & $<0.0001$ \\
\hline No_rank & -0.0998 & 0.0476 & $<0.0001$ & -0.099 & 0.0354 & 0.001 & -0.1423 & 0.05 & $<0.0001$ \\
\hline
\end{tabular}

tion strategies dropped to $17 \%$ in the RS + biochar (from $27 \%$ in the RS), while the percentage of sensitive species increased from $40 \%$ in the RS to $50 \%$ in the RS + biochar (Fig. 3). In addition, life strategies varied among the dominant phyla, while some phyla exhibited similar distributions under the three treatments (Fig. 4).

\subsection{Network analysis of fungal communities}

We explored fungal co-occurrence patterns among the three different treatments using network analyses based on significant $(p<0.05)$ correlations of abundances. Due to the complexity of the networks, only a very limited number of key nodes with higher connectivities were considered. Key nodes fundamentally structure networks, and networks would look significantly different without their presence (Agler et al., 2016). The positions of the key nodes within the networks according to our criteria (Fig. 5) are indicated in Fig. 6(A)-1, $(B)-1$, and (C)-1. The heat map highlights trends in the abundances of taxa representing key nodes (Fig. 6(A)-2, (B)-2 and (C)-2). Based on the reconstructed networks, detailed network topological analyses were then performed (Table 2). Notably, there were more positive than negative correlations in all sub-networks, regardless of treatments. Additionally, analysis of network topological properties indicated that the $\mathrm{RS}+$ biochar increased the number of positive correlations among key nodes. Although many of the fungi that were identified in this study could not be identified taxonomically, the key nodes representing fungal OTUs belonged to some identifiable functional guilds (Table S1). Of these fungi, the abundance of a dung saprotroph in the genus Schizothecium (OTU138) increased over time. However, many of the unidentified fungi (e.g., OTU120, OTU217, OTU266, OTU327, OTU335, OTU338, OTU404, OTU405) were key nodes in the networks of each treatment. Further BLAST-based identifications are provided for key nodes in Table S2, and were used to calculate a consistency index.

\subsection{Relationship between fungal communities and environmental variables}

Mantel and partial Mantel tests were performed to determine 

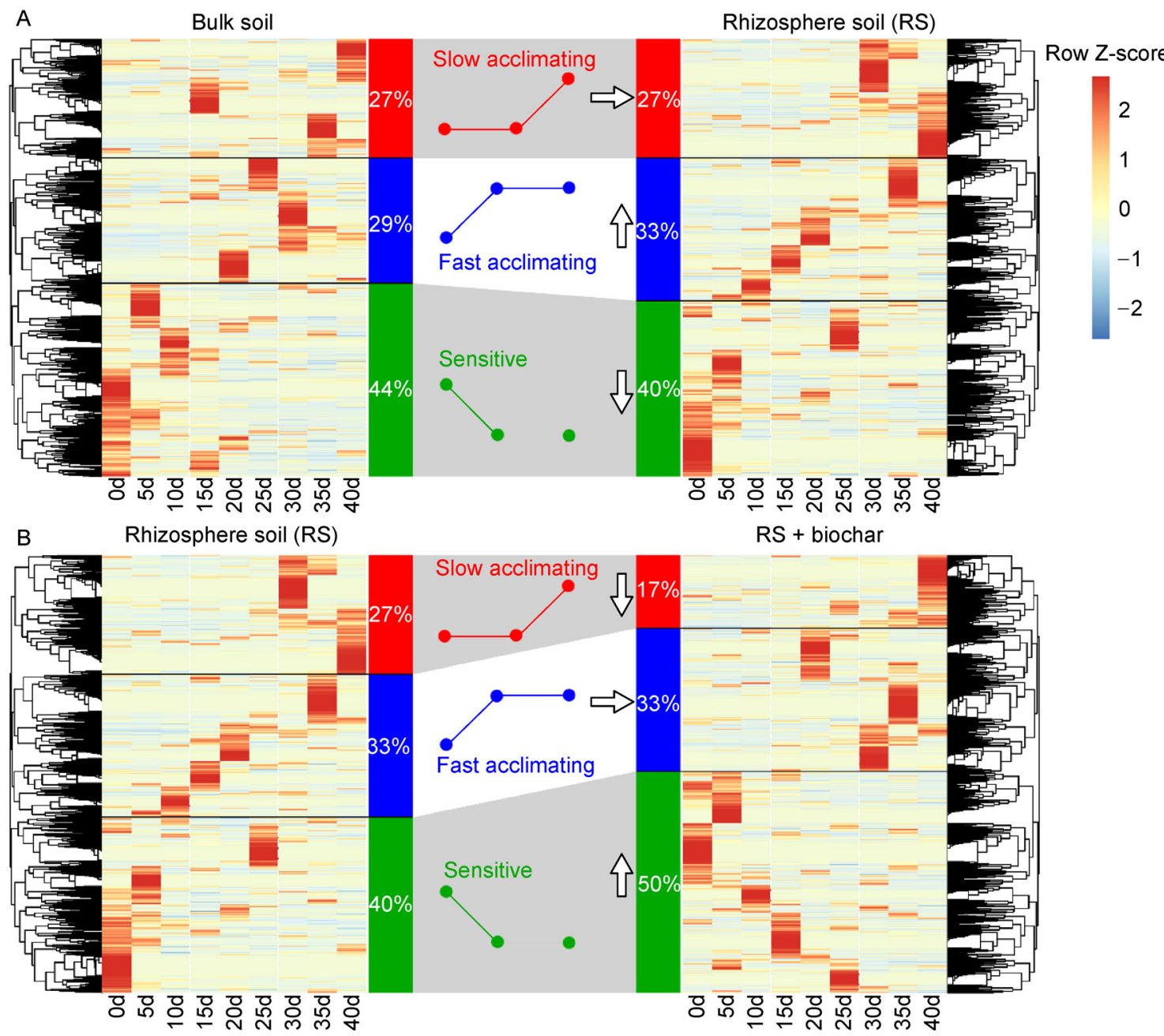

Fig. 3 Heat maps showing the relative abundance of each fungal operational taxonomic unit (OTU) (rows) at three time points (earlier stage $(0-10 \mathrm{~d})$, middle stage (15-25 d), and later stage (30-40 d)) in different treatments. The responses over time were classified into life strategies (the given labels in the center) using cluster analysis dendrograms, as shown on the outside of each heat map. The proportion of species that employed each life strategy (coloured bars, white percentage) differed in the fungal communities from different treatments. (A) The heat map on the left is the bulk soil and the one on the right is the rhizosphere soil (RS); (B) the heat map on the left is the rhizosphere soil (RS), the one on the right is the RS + biochar.

how environmental factors including the soil geochemical variables $\left(\mathrm{pH}, \mathrm{NO}_{3}{ }^{-} \mathrm{N}, \mathrm{NH}_{4}{ }^{+} \mathrm{N}\right.$, available phosphorous, trace elements, and soil surface structural parameters), ryegrass roots, and antibiotics affected fungal community composition during the 40-day experiment (Table S3). Soil geochemical variables were the most important factors related to differences in fungal $\beta$-diversity among all samples, and especially during the earlier stages of the experiment $\left(r_{\mathrm{m}}=0.334\right.$, $p<0.01$ ). Furthermore, we found that fungal community composition in the RS was primarily affected by soil geochemical variables, whereas community composition in the RS + biochar samples was mostly influenced by ryegrass roots. Furthermore, correlation analysis between antibiotics and fungal taxa indicated that lomefloxacin, ciprofloxacin, and norfloxacin were negatively correlated with unclassified fungal abundances $(p<0.01)$, but positively associated with Asco- mycota abundances $(p<0.05$; Table S4). OTU richness overall was negatively correlated with quinolones (enrofloxacin, lomefloxacin, ciprofloxacin, and norfloxacin), and Basidiomycota abundances were significantly negatively correlated to sulfamethazine concentrations $(p<0.05)$.

We conducted structural equation model (SEM) in order to determine the effects of ryegrass roots, soil $\mathrm{pH}$, soil trace elements, soil structure and antibiotics on fungal life strategies (i.e., OTU abundances) and interactions (positive/total network edges of the key nodes) (Fig. 7). The SEM demonstrated that the influence of ryegrass roots on fungal life strategies and interactions in the RS was mediated by soil $\mathrm{pH}(r=$ $-0.374, p=0.004)$, soil structure $(r=0.625, p<0.001)$, and antibiotics $(r=-0.596, p<0.001)$. As the same time, ryegrass roots had positive direct impacts on fungal life strategies $(r=$ $0.212, p=0.047)$ and interactions $(r=0.241, p=0.039)$. In 


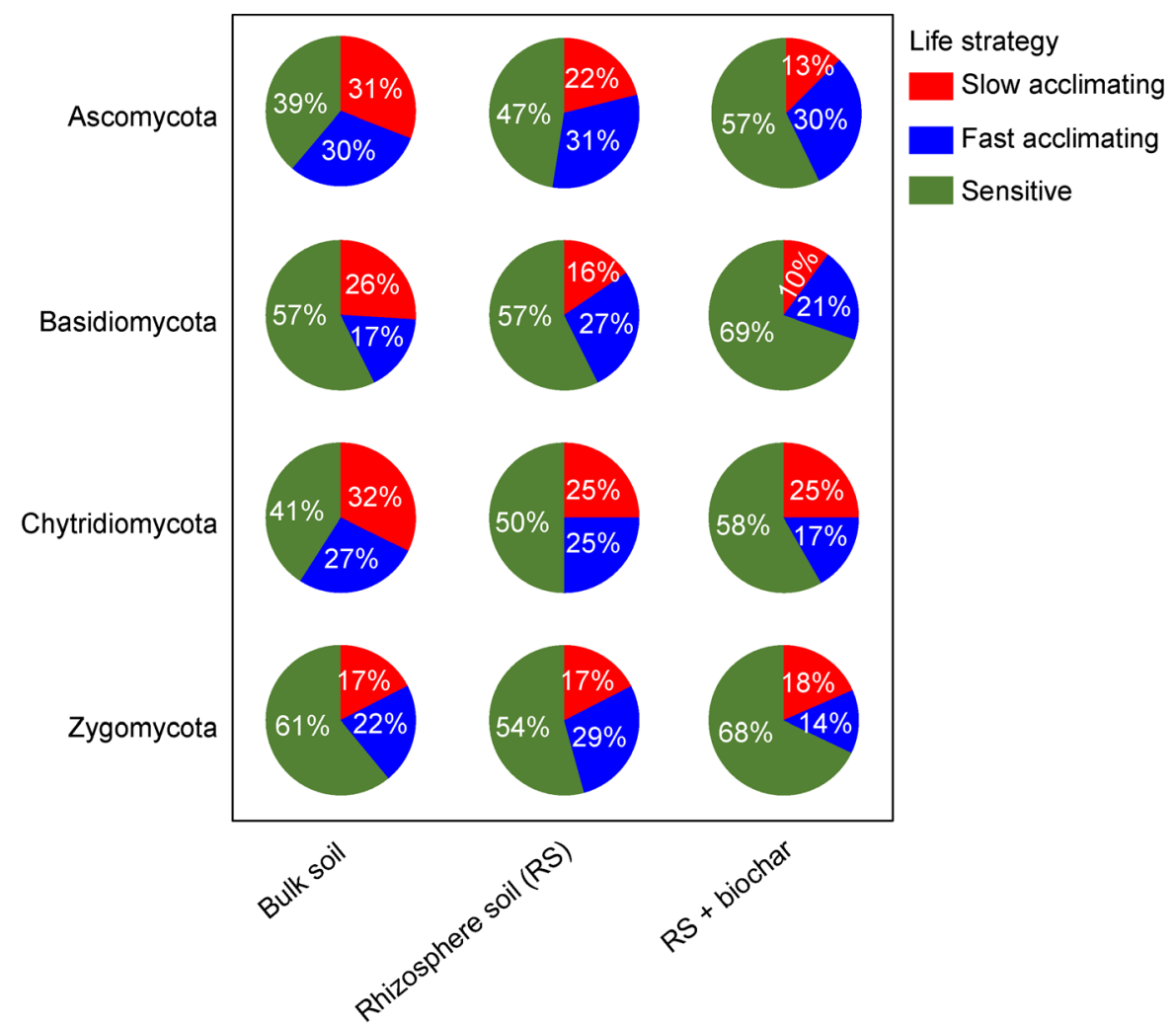

Fig. 4 Pie charts showing the distribution of life strategy within the most dominant phyla among three treatments.

A Residual plot with outliers, Method II normal distribution, $R^{2}=0.7866$

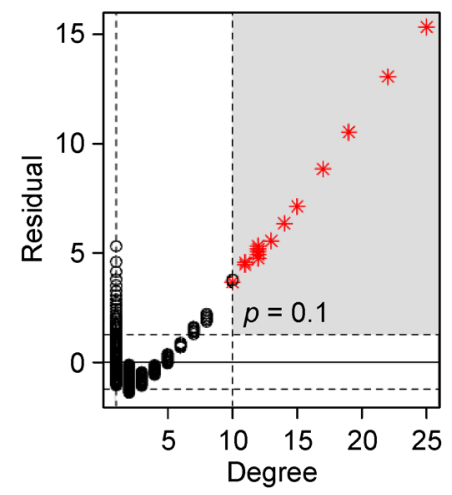

B Residual plot with outliers, Method II normal distribution, $R^{2}=0.7301$

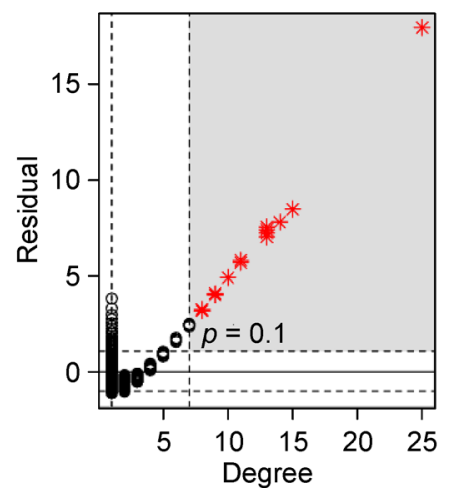

C Residual plot with outliers, Method II normal distribution, $R^{2}=0.7808$

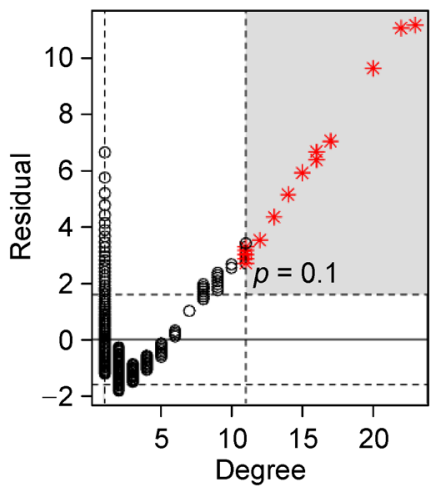

Fig. 5 Criteria for defining key nodes in fungal species interaction networks. The red dots represented the key nodes. (A) The bulk soil; (B) the rhizosphere soil (RS); and (C) the RS + biochar.

addition, the strongest relationship observed in the SEM analysis was between soil $\mathrm{pH}$ and fungal life strategies $(r=$ $0.753, p<0.001$ ). For the RS + biochar, the positive impact of ryegrass roots on soil trace elements $(r=0.893, p<0.001)$ and soil structure $(r=0.966, p<0.001)$ were stronger in the $\mathrm{RS}+$ biochar than in RS, but the direct impact on fungal interactions $(r=-0.048, p=0.731)$ was decreased. Moreover, the impact of soil $\mathrm{pH}$ on antibiotics and fungal interactions significantly increased $(p<0.01)$, but the impact on fungal life strategies significantly decreased $(r=0.194, p=0.262)$. Soil
$\mathrm{pH}$ was the most significant positive parameter associated with fungal life strategies and interactions, as indicated by the standardized total effects from the SEM.

\section{Discussion}

4.1 Faster temporal turnover of fungal communities

Temporal turnover, as measured by time-decay relationships, is an important indicator of the successional dynamics of 

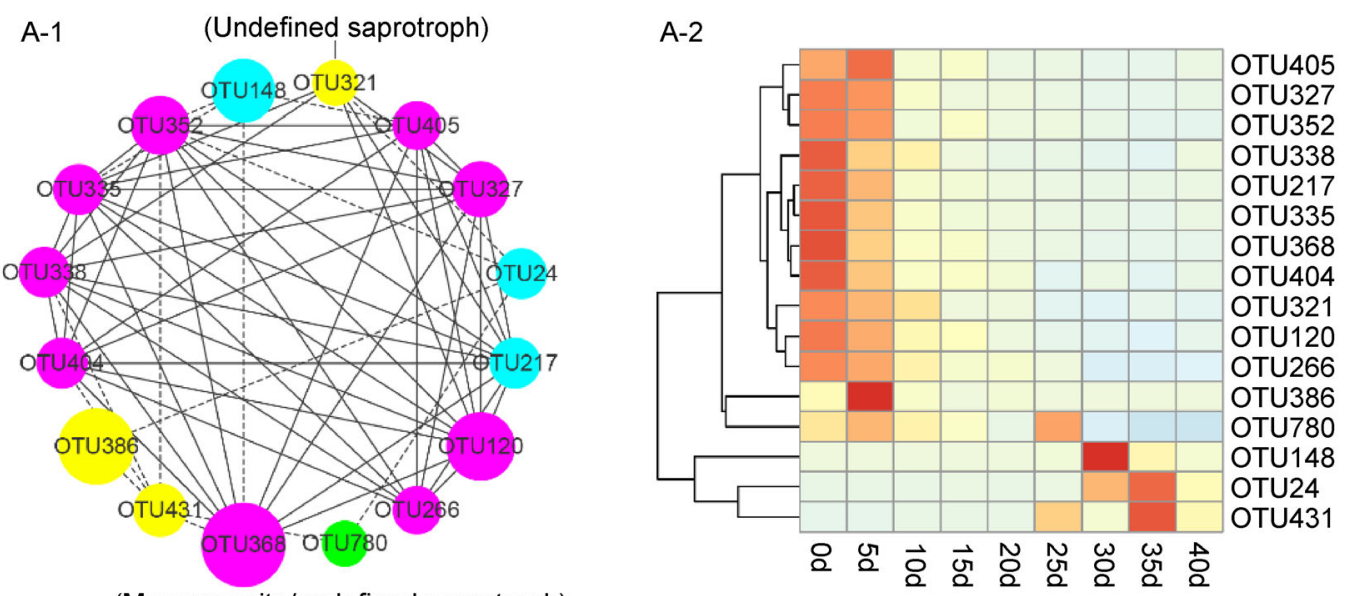

(Mycoparasite/undefined saprotroph)

B-2

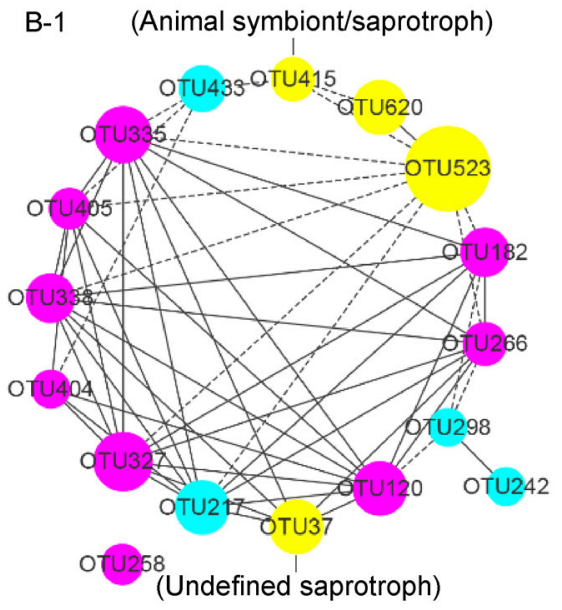

C-1 (Dung saprotroph/wood saprotroph)

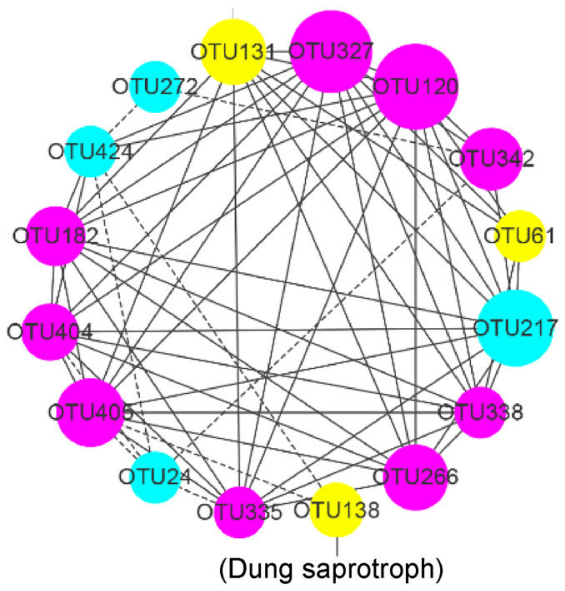

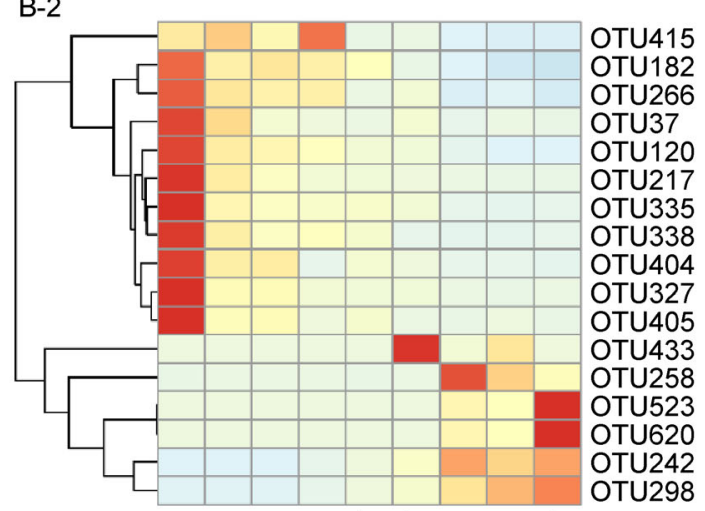

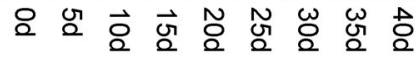

C-2

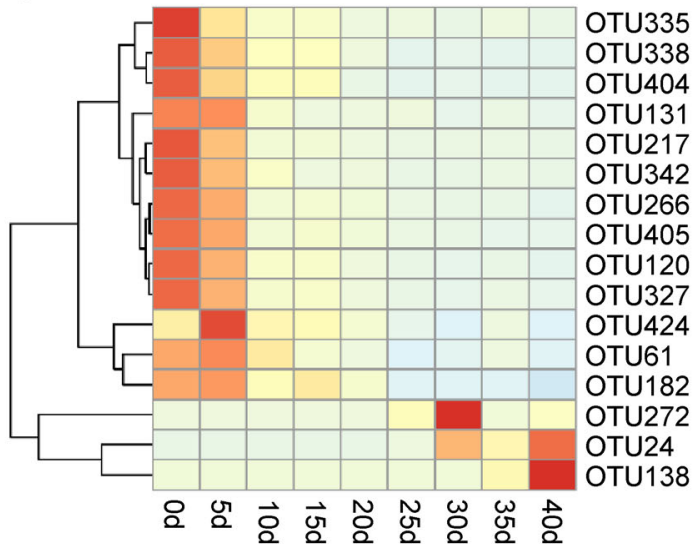

Row Z-score

2

1

0

$-1$

$-2$

Nodes

Ascomycota

- Basidiomycota

Unclassified_fungi

NA

Edges

Positive
N......... Negative

Fig. 6 The network and heat map of the key nodes in the bulk soil (A), rhizosphere soil (RS) (B) and RS + biochar (C). Identifiable functional guilds were labelled. Each node indicates the OTU of the fungi. The size of each node is proportional to the number of connections (that is, the degree). The edge between two nodes indicates the positive (solid) or negative (dash) interactions. 
Table 2 Statistics and topological parameters for the key nodes from fungal networks.

\begin{tabular}{llll}
\hline & Bulk soil & Rhizosphere soil (RS) & RS + biochar \\
\hline Number of nodes & 16 & 17 & 16 \\
Number of co-presences & 48 & 38 & 56 \\
Number of mutual-exclusions & 14 & 16 & 9 \\
Mean number of neighbors & 7.75 & 6.353 & 8.125 \\
Clustering coefficient & 0.629 & 0.604 & 0.621 \\
Network density & 0.517 & 0.397 & 0.542 \\
Network heterogeneity & 0.409 & 0.529 & 0.399 \\
Connected components & 1 & 2 & 1 \\
Network centralization & 0.324 & 0.258 & 0.295 \\
\hline
\end{tabular}

A-1

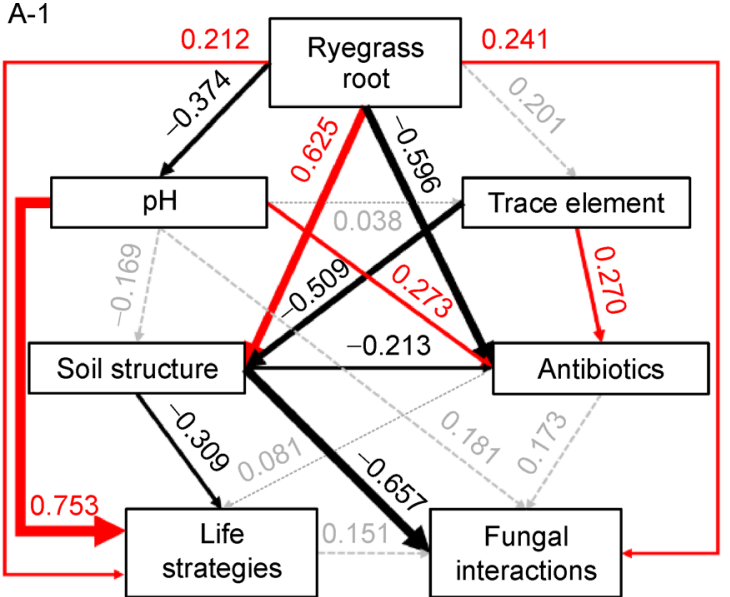

$\chi^{2}=1.817, P=0.403, \mathrm{df}=2 ;$ bootstrap $P=0.348$;

RMSEA $=0.000, P=0.447$

B-1

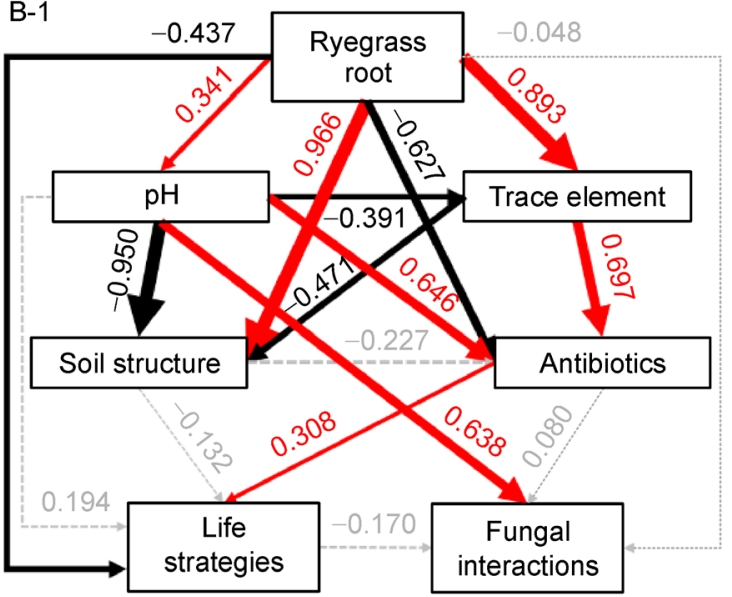

$\chi^{2}=1.137, P=0.768, \mathrm{df}=3$; bootstrap $P=0.453$;

RMSEA $=0.000, P=0.800$
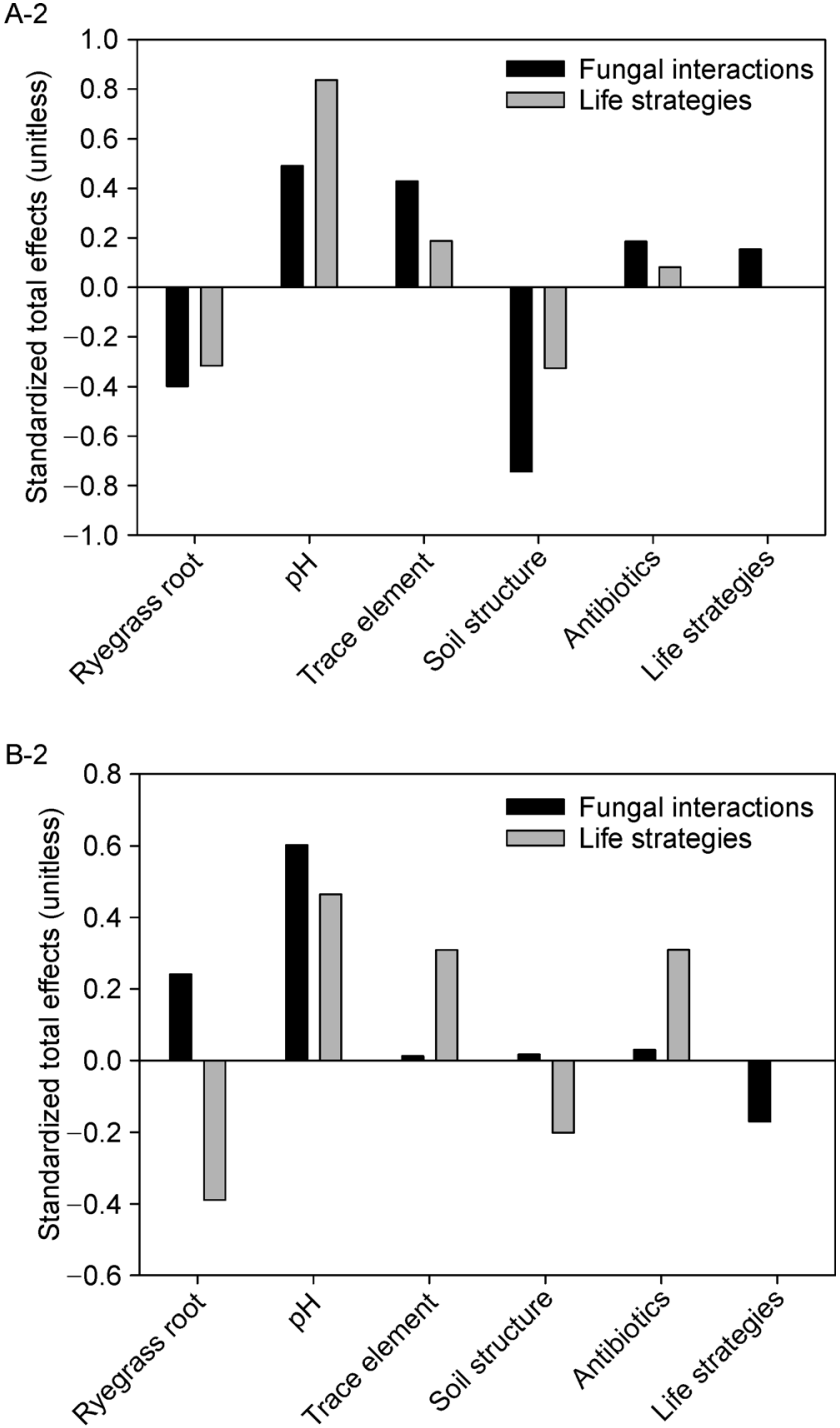

Fig. 7 Structural equation models of the ryegrass roots, soil pH, soil trace elements, soil structure and antibiotics as predictors of fungal life strategies and interactions in the rhizosphere soil (RS) (A) and RS + biochar (B). Solid red arrows represent positive paths $(p<0.05)$, solid black arrows represent negative paths $(p<0.05)$ and dotted grey arrows represent non-significant paths $(p>0.05)$. The line width is proportional to the $r$ value. 
biological communities (Liang et al., 2015). Species temporal turnover is defined as the number of species that are eliminated and replaced per unit time (Hatosy et al., 2013), and the slope of a linear regression model of logarithmically transformed community similarity and time can be used as a measure of temporal turnover (Nekola and White, 1999; Korhonen et al., 2010). Here, we showed that similarity in fungal community composition significantly declines linearly over time in bulk soil, RS, and RS + biochar $(p<0.0001$; Fig. 2). These results strongly supported the time-decay relationship in ecological theory (Liang et al., 2015).

We further observed that biochar addition promoted the succession of fungal communities $(p<0.0001)$. One explanation for this effect could be that the biochar addition modulates environmental conditions, thereby affecting the composition and structure of the fungal communities (Fig. 1 and Fig. S1). Biochar accelerates the growth and proliferation of soil fungi and affects the structure and function of soil fungal communities by altering soil physical and chemical properties, such as soil resource reserves (e.g., available carbon, nutrient substances, moisture) and physicochemical components (e.g., pH, cation exchange capacity) (Zhu et al., 2017). In support of this explanation, significant positive correlations have been reported between fungal $\alpha$-diversity and $\beta$ diversity, which indicates compositional and structural variations in fungal communities that directly affects their succession rates (Pereira e Silva et al., 2012). An alternative explanation for our observations is that biochar addition boosts the growth of ryegrass roots. After biochar addition, ryegrass roots became the primary factor that affects fungal communities ( $r=0.428, p<0.05$; Table S3). It has been observed that biochar addition benefits the growth of ryegrass in antibiotic-contaminated soils (Liang et al., 2017), and this stimulation may result from complex ecological interactions. For instance, biochar can modify the relationship between inter-rhizosphere microbes and plants and also affect competition between beneficial soil microbes and soiltransmitted pathogens, which then triggers plant system defenses against soil-transmitted pathogens. Elad et al. (2010) found that the application of biochar successfully induced resistance to two foliar fungal pathogens (Botrytis cinerea and Leveillula taurica) in both pepper and tomato plants. The stimulation of these defenses could be mediated by either enhanced interaction between plant roots and rhizosphere fungi that promote plant growth or, alternatively, by direct toxic effects of biochar components on microbial pathogens. Graber et al. (2010) demonstrated that some compounds in biochar, like volatile organic compounds, are microbial inhibitors that can induce toxicity directly to soil microbial pathogens, thereby benefitting plant growth. In addition to the above mechanisms, ryegrass roots indirectly affect successional rates of fungal communities via root secretion of compounds (Song et al., 2016). The dependence of all the above temporal changes on particular environmental stresses or rather, whether they change randomly with succession, requires further investigation.

\subsection{Alternation of soil fungi life strategies}

We found that more significant changes occurred in the life strategies of soil fungi in RS + biochar than compared to soils with the RS (Fig. 3). Based on variation in the relative richness of fungal groups, three life strategies were determined: slow acclimating, fast acclimating, and sensitive. This conceptual framework helps predict key lifestyle traits for major taxa and also better understand microbial influence on soil processes (Fierer, 2017). The proportion of sensitive fungi increased and the proportion of slow acclimating fungi decreased in RS + biochar. These differences may be due to different physiologic characteristics of fungi in the two treatments and differences in their sensitivity toward environmental variation induced by the treatments. Consequently, these results suggested that the fungal community in RS + biochar may be more sensitive to environmental changes than those within the bulk soil or RS. Paz-Ferreiro et al. (2013) demonstrated that some components in biochar may affect the activities of fungi and reshape soil fungal communities, indicating that soils added with biochar promote the growth of some fungal groups. Additionally, the shift in life strategies may be partially due to a withinspecies change in ecological strategy (Table S5). Evans and Wallenstein (2014) noted that changes in the distribution of a community's ecological strategies may occur through a structural shift in the community (such as colonization by new species, increase in abundances, or out compete other species) or through physiologic shifts in individual species. In contrast, Wang et al. (2011) suggested that shifts in ecological strategies may be independent of variation in community composition, and could occur at different rates, indicating that variation in ecological characteristics controlled by different factors. Thus, whether the shift in ecological strategies observed here is caused by shifts in species composition or by other factors remains unclear. Further studies are needed to evaluate the processes that are involved in within-species changes in ecological strategies in addition to phylum-level responses.

Fungi are more capable of easily degrading refractory carbon within biochar and that fungi can grow more effectively in biochar pores and utilize additional resources than bacteria (Lehmann et al., 2011). Consequently, biochar addition could improve fungal richness and increase the fungal/bacteria ratio of soils. Warnock et al. (2007) demonstrated that biochar addition to soils enhances the ability of fungi to proliferate in the roots of crops and changes microbial community. Moreover, fungal communities are regulated by the type and availability of resources (Moll et al., 2015). One comprehensive study showed that fungal community diversity is significantly correlated with the distribution of several enriched fungal groups and the carbon content of soils (Liu et al., 2015). Fungal groups differ in their abilities to utilize resources, and some of them target specific organic compounds (Sun et al., 2016), which results in the occupation of different niches according to available carbon sources. In the unique rhizosphere environment, plant roots constantly secrete consider- 
able amounts of organic substances into the soils to form rhizosphere sediments. Plants thus regulate the type and quantity of soil fungi that surround roots by modifying the nutrition and energy sources to rhizosphere fungi and also regulate the interactions in the rhizosphere between plants and fungi (Singh and Mukerji, 2006). As demonstrated here, the abilities of fungi to utilize resources differs and contributes to an inherent balance in resource distribution among different treatments. Thus, variations in fungal life strategies are affected by biochar addition.

\subsection{Increased interactions among soil fungi}

We constructed a molecular ecological network to assess interactions among soil fungi in three different treatments. Key nodes that are determined by connectedness are considered to be species that play important roles in their communities (Wang et al., 2017). The absence of these nodes leads to a lack of network connectivity, and greatly affects the topological structure of the whole community network. Most of the key nodes identified here are saprophytic fungi belonging to Ascomycota and Basidiomycota phyla, which is consistent with results from Rudnick et al. (2015). Saprophytic fungi, which inhabit the rhizosphere, are largely distributed in three phyla: Zygomycota, Ascomycota, and Basidiomycota. Saprophytic fungi can control the cycling rate of nutrients in agricultural ecosystems and promote the decomposition of organic matrices (Toju et al., 2016). Solaiman et al. (2010) found that saprophytic fungi are convenient to grow in biochar due to their hyphal structures and highly active exoenzymes. Further, biochar provides protection for saprophytic fungi, promotes the symbiosis of fungi and roots, and promotes the growth of fungal mycorrhizae.

Biochar amendment increased the active relationships between populations while reducing passive relationships, as compared to the bulk soil and RS (Table 2). This phenomenon may be related to the porosity and surface characteristics of biochar that provide a good habitat for the growth and proliferation of soil fungi and also reduces competition between fungi (Lehmann et al., 2011). Quilliam et al. (2013) demonstrated that biochar pores can effectively protect fungi against competitors. Further, Abel et al. (2013) indicated that biochar can improve the habitat for microbes by improving the physical properties of soils, and promote cooperative relationships among microorganisms, that then allow for good utilization of soil resources (Toju et al., 2016). In addition to soil $\mathrm{pH}$ was found to significantly influence fungal interactions in $\mathrm{RS}+$ biochar $(p<0.001$; Fig. 7). Changes in environmental properties like $\mathrm{pH}$ and temperature alter microbial ecological networks (Deng et al., 2012; Shi et al., 2016). However, the specific contribution of various factors to the overall interaction network still requires further experimental verification (Jiang et al., 2017).
4.4 Alternative mechanisms influencing fungal life strategies and interactions

We used structural equation models to assess the contributions of various factors to the fungal community compositions (Fig. 7). Results from these analyses indicated that ryegrass roots affect the life strategies and interactions of fungi through their own direct and indirect effects on soil $\mathrm{pH}$, trace elements, soil structures, and antibiotics. Biochar addition enhanced the positive effect of soil $\mathrm{pH}$ in deceasing antibiotic concentrations $(p<0.01)$, indicating that $\mathrm{pH}$ variation may promote the degradation of antibiotics (Liang et al., 2017). Liang et al. (2017) found that a combination of biochar and ryegrass treatment mitigates the effects of antibiotics more effectively than with ryegrass planting alone. Following these observation, the rapid mitigation of antibiotics in the $\mathrm{RS}+$ biochar treatment significantly enhanced fungal inter-specific relationships and increased the number of positive correlations among fungal taxa abundances. In addition, Mantel and partial Mantel tests suggested that the physical and chemical properties of soil and ryegrass roots are the primary parameters affecting fungal communities in RS + biochar (Table S3). Our findings coincide with previous research, which show that soil $\mathrm{pH}$ and nutrient levels are the best predictive factors of soil fungal community properties (Lauber et al., 2008; Rousk et al., 2010). Variation of microbial diversity in soil may be regulated through the supply of organic matter nutrients (Cookson et al., 2005; Housman et al., 2007). The secretions of plant roots can also affect microbial communities through the provision of available carbon sources (Song et al., 2016).

The mechanisms underlying how plants and soil amendments impact bacterial and fungal diversity have been proposed recently (Xu et al., 2014; Ducey et al., 2015). The current study provided a significant step forward in improving our understanding of the factors that influence rhizosphere fungal communities in manure fertilized soils. It should be noted that our laboratory-based experiments may not necessarily reflect what would happen under field conditions. Consequently, further experiments are needed in the field.

\section{Acknowledgments}

This work was supported by Strategic Priority Research Program (B) of the Chinese Academy of Sciences (XDB15010100), the National Natural Science Foundation of China (No. 41622104, 41877060), Distinguished Young Scholar Program of the Jiangsu Province (BK20160050), and Youth Innovation Promotion Association of Chinese Academy of Sciences (2016284).

\section{Conflict of interest}

The authors declare no conflict of interest. 


\section{Electronic supplementary material}

Supplementary material is available in the online version of this article at http://dx.doi.org/10.1007/042832-0008-8 and is accessible for authorized users.

\section{References}

Abel, S., Peters, A., Trinks, S., Schonsky, H., Facklam, M., Wessolek, G., 2013. Impact of biochar and hydrochar addition on water retention and water repellency of sandy soil. Geoderma 202-203, 183-191.

Agler, M.T., Ruhe, J., Kroll, S., Morhenn, C., Kim, S.T., Weigel, D., Kemen, E.M., 2016. Microbial hub taxa link host and abiotic factors to plant microbiome variation. PLoS Biology 14, e1002352.

Ameloot, N., Neve, S.D., Jegajeevagan, K., Yildiz G., Buchan D. Funkuin Y.N., Prins W., Bouckaert L., Sleutel S., 2013. Short-term $\mathrm{CO}_{2}$ and $\mathrm{N}_{2} \mathrm{O}$ emissions and microbial properties of biochar amended sandy loam soils. Soil Biology \& Biochemistry 57, 401410.

Cookson, W.R., Abaye, D.A., Marschner, P., Murphy, D.V., Stockdale, E.A., Goulding, K.W.T., 2005. The contribution of soil organic matter fractions to carbon and nitrogen mineralization and microbial community size and structure. Soil Biology \& Biochemistry $37,1726-1737$.

Deng, Y., Jiang, Y.H., Yang, Y., He, Z., Luo, F., Zhou, J., 2012. Molecular ecological network analyses. BMC Bioinformatics 13, 113.

Dickie, I.A., Cooper, J.A., Bufford, J.L., Hulme, P.E., Bates, S.T., 2016. Loss of functional diversity and network modularity in introduced plant-fungal symbioses. AoB Plants 9, plw084.

Ducey, T., Novak, J., Johnson, M., 2015. Effects of biochar blends on microbial community composition in two coastal plain soils. Agriculture 5, 1060-1075.

Durenkamp, M., Luo, Y., Brookes, P.C., 2010. Impact of black carbon addition to soil on the determination of soil microbial biomass by fumigation extraction. Soil Biology \& Biochemistry 42, 2026-2029.

Elad, Y., David, D.R., Harel, Y.M., Borenshtein, M., Kalifa, H.B., Silber, A., Graber, E.R., 2010. Induction of systemic resistance in plants by biochar, a soil-applied carbon sequestering agent. Phytopathology 100, 913-921.

Evans, S.E., Wallenstein, M.D., 2014. Climate change alters ecological strategies of soil bacteria. Ecology Letters 17, 155-164.

Fierer, N., 2017. Embracing the unknown: disentangling the complexities of the soil microbiome. Nature Reviews. Microbiology 15, 579-590.

Fuhrman, J.A., 2009. Microbial community structure and its functional implications. Nature 459, 193-199.

Gilbert, J.A., Steele, J.A., Caporaso, J.G., Steinbrück, L., Reeder, J., Temperton, B., Huse, S., McHardy, A.C., Knight, R., Joint, I., Somerfield, P., Fuhrman, J.A., Field, D., 2012. Defining seasonal marine microbial community dynamics. ISME Journal 6, 298-308.

Graber, E.R., Harel, Y.M., Kolton, M., Cytryn E., Silber A., David D.R., Tsechansky L., Borenshtein M., Elad Y., 2010. Biochar impact on development and productivity of pepper and tomato grown in fertigated soilless media. Plant and Soil 337, 481-496.

Grime, J.P., 1977. Evidence for the existence of three primary strategies in plants and its relevance to ecological and evolutionary theory. American Naturalist 111, 1169-1194.

Hammesfahr, U., Heuer, H., Manzke, B., Smalla, K., Thiele-Bruhn, S., 2008. Impact of the antibiotic sulfadiazine and pig manure on the microbial community structure in agricultural soils. Soil Biology \& Biochemistry 40, 1583-1591.

Hatosy, S.M., Martiny, J.B., Sachdeva, R., Steele, J., Fuhrman, J.A., Martiny, A.C., 2013. Beta diversity of marine bacteria depends on temporal scale. Ecology 94, 1898-1904.

Holland, M.D., Hastings, A., 2008. Strong effect of dispersal network structure on ecological dynamics. Nature 456, 792-794.

Housman, D.C., Yeager, C.M., Darby, B.J., Sanford, R.L. Jr, Kuske, C. R., Neher, D.A., Belnap, J., 2007. Heterogeneity of soil nutrients and subsurface biota in a dryland ecosystem. Soil Biology \& Biochemistry 39, 2138-2149.

Jia, M., Wang, F., Bian, Y., Stedtfeld, R.D., Liu, G., Yu, J., Jiang, X., 2018. Sorption of sulfamethazine to biochars as affected by dissolved organic matters of different origin. Bioresource Technology 248, 36-43.

Jiang, Y., Liu, M., Zhang, J., Chen, Y., Chen, X., Chen, L., Li, H., Zhang, X.X., Sun, B., 2017. Nematode grazing promotes bacterial community dynamics in soil at the aggregate level. ISME Journal 11, 2705-2717.

Korhonen, J.J., Soininen, J., Hillebrand, H., 2010. A quantitative analysis of temporal turnover in aquatic species assemblages across ecosystems. Ecology 91, 508-517.

Lauber, C.L., Strickland, M.S., Bradford, M.A., Fierer, N., 2008. The influence of soil properties on the structure of bacterial and fungal communities across land-use types. Soil Biology \& Biochemistry 40, 2407-2415.

Lehmann, J., Rillig, M.C., Thies, J., Masiello, C.A., Hockaday, W.C., Crowley, D., 2011. Biochar effects on soil biota - A review. Soil Biology \& Biochemistry 43, 1812-1836.

Liang, Y., Jiang, Y., Wang, F., Wen, C., Deng, Y., Xue, K., Qin, Y., Yang, Y., Wu, L., Zhou, J., Sun, B., 2015. Long-term soil transplant simulating climate change with latitude significantly alters microbial temporal turnover. ISME Journal 9, 2561-2572.

Liang, Y., Pei, M., Wang, D., Cao, S., Xiao, X., Sun, B., 2017. Improvement of soil ecosystem multifunctionality by dissipating manure-induced antibiotics and resistance genes. Environmental Science \& Technology 51, 4988-4998.

Liu, J., Sui, Y., Yu, Z., Shi, Y., Chu, H., Jin, J., Liu, X., Wang, G., 2015. Soil carbon content drives the biogeographical distribution of fungal communities in the black soil zone of northeast China. Soil Biology \& Biochemistry 83, 29-39.

Moll, J., Goldmann, K., Kramer, S., Hempel, S., Kandeler, E., Marhan, S., Ruess, L., Krüger, D., Buscot, F., 2015. Resource type and availability regulate fungal communities along arable soil profiles. Microbial Ecology 70, 390-399.

Nekola, J.C., White, P.S., 1999. The distance decay of similarity in biogeography and ecology. Journal of Biogeography 26, 867-878.

Nguyen, N.H., Song, Z., Bates, S.T., Branco, S., Tedersoo, L., Menke, J., Schilling, J.S., Kennedy, P.G., 2016. FUNGuild: An open annotation tool for parsing fungal community datasets by 
ecological guild. Fungal Ecology 20, 241-248.

Op De Beeck, M., Lievens, B., Busschaert, P., Declerck, S., Vangronsveld, J., Colpaert, J.V., 2014. Comparison and validation of some ITS primer pairs useful for fungal metabarcoding studies. PLoS One 9, e97629.

Paz-Ferreiro, J., Fu, S., Méndez, A., Gascó, G., 2013. Interactive effects of biochar and the earthworm Pontoscolex corethrurus on plant productivity and soil enzyme activities. Journal of Soils and Sediments 14, 483-494.

Pereira e Silva, M.C., Dias, A.C., van Elsas, J.D., Salles, J.F., 2012. Spatial and temporal variation of archaeal, bacterial and fungal communities in agricultural soils. PLoS One 7, e51554.

Quilliam, R.S., Glanville, H.C., Wade, S.C., Jones, D.L., 2013. Life in the "charosphere" - does biochar in agricultural soil provide a significant habitat for microorganisms? Soil Biology \& Biochemistry 65, 287-293.

Rousk, J., Bååth, E., Brookes, P.C., Lauber, C.L., Lozupone, C., Caporaso, J.G., Knight, R., Fierer, N., 2010. Soil bacterial and fungal communities across a $\mathrm{pH}$ gradient in an arable soil. ISME Journal 4, 1340-1351.

Rudnick, M.B., van Veen, J.A., de Boer, W., 2015. Baiting of rhizosphere bacteria with hyphae of common soil fungi reveals a diverse group of potentially mycophagous secondary consumers. Soil Biology \& Biochemistry $88,73-82$.

Shade, A., Caporaso, J.G., Handelsman, J., Knight, R., Fierer, N., 2013. A meta-analysis of changes in bacterial and archaeal communities with time. ISME Journal 7, 1493-1506.

Shi, S., Nuccio, E.E., Shi, Z.J., He, Z., Zhou, J., Firestone, M.K., 2016. The interconnected rhizosphere: High network complexity dominates rhizosphere assemblages. Ecology Letters 19, 926-936.

Singh, G., Mukerji, K.G., 2006. Root exudates as determinant of rhizospheric microbial biodiversity. In: Mukerji K.G., Manoharachary C., Singh J. (eds.). Microbial Activity in the Rhizosphere. Heidelberg: Springer, 39-53.

Solaiman, Z.M., Blackwell, P., Abbott, L.K., Storer, P., 2010. Direct and residual effect of biochar application on mycorrhizal root colonization, growth and nutrition of wheat. Australian Journal of Soil Research 48, 546-554.

Song, Y., Li, Y., Zhang, W., Wang, F., Bian, Y., Boughner, L.A., Jiang, X., 2016. Novel biochar-plant tandem approach for remediating hexachlorobenzene contaminated soils: proof-of-concept and new insight into the rhizosphere. Journal of Agricultural and Food Chemistry 64, 5464-5471.

Steinbeiss, S., Gleixner, G., Antonietti, M., 2009. Effect of biochar amendment on soil carbon balance and soil microbial activity. Soil Biology \& Biochemistry 41, 1301-1310.

Sun, R., Dsouza, M., Gilbert, J.A., Guo, X., Wang, D., Guo, Z., Ni, Y., Chu, H., 2016. Fungal community composition in soils subjected to long-term chemical fertilization is most influenced by the type of organic matter. Environmental Microbiology 18, 5137-5150.

Toju, H., Kishida, O., Katayama, N., Takagi, K., 2016. Networks depicting the fine-scale co-occurrences of fungi in soil horizons. PLoS One 11, e0165987.

Wang, H., Wei, Z., Mei, L., Gu, J., Yin, S., Faust, K., Raes, J., Deng, Y., Wang, Y., Shen, Q., Yin, S., 2017. Combined use of network inference tools identifies ecologically meaningful bacterial associations in a paddy soil. Soil Biology \& Biochemistry 105, 227-235.

Wang, S., Spor, A., Nidelet, T., Montalent, P., Dillmann, C., de Vienne, D., Sicard, D., 2011. Switch between life history strategies due to changes in glycolytic enzyme gene dosage in Saccharomyces cerevisiae. Applied and Environmental Microbiology 77, 452-459.

Warnock, D.D., Lehmann, J., Kuyper, T.W., Rillig, M.C., 2007. Mycorrhizal responses to biochar in soil - concepts and mechanisms. Plant and Soil 300, 9-20.

Xiao, X., Liang, Y., Zhou, S., Zhuang S., Sun B., 2017. Fungal community reveals less dispersal limitation and potentially more connected network than that of bacteria in bamboo forest soils. Molecular Ecology 27, 550-563.

Xu, H.J., Wang, X.H., Li, H., Yao, H.Y., Su, J.Q., Zhu, Y.G., 2014. Biochar impacts soil microbial community composition and nitrogen cycling in an acidic soil planted with rape. Environmental Science \& Technology 48, 9391-9399.

Yamato, M., Okimori, Y., Wibowo, I.F., Anshori, S., Ogawa, M., 2006. Effects of the application of charred bark of Acacia mangiumon the yield of maize, cowpea and peanut, and soil chemical properties in South Sumatra, Indonesia. Soil Science and Plant Nutrition 52, 489495.

Zhu, X., Chen, B., Zhu, L., Xing, B., 2017. Effects and mechanisms of biochar-microbe interactions in soil improvement and pollution remediation: A review. Environ Pollut 227, 98-115. 